\title{
A Progressive Algorithm For Three Point Transport
}

Reynald Dumont, Kadi Bouatouch, Philippe Gosselin

\section{$\mathbf{N}^{\circ} 3305$}

Novembre 1997

THÈME 3 



\title{
A Progressive Algorithm For Three Point Transport
}

\author{
Reynald Dumont, Kadi Bouatouch, Philippe Gosselin \\ Thème 3 - Interaction homme-machine, \\ images, données, connaissances \\ Projet SIAMES \\ Rapport de recherche $\quad 3305 \quad$ Novembre $1997 \quad 17$ pages
}

\begin{abstract}
When computing global illumination in environments made up of surfaces with general Bidirectionnal Reflection Distribution Function, a three point formulation of the rendering equation can be used. Brute-force algorithms can lead to a linear system of equations whose matrix is cubic, which is expensive in time and space. The hierarchical approach is more efficient. Aupperle et al. proposed a hierchical three point algorithm to compute global illumination in presence of glossy reflection. We present in this paper some improvements we brought to this method: shooting, "lazy" push-pull, photometric subdivision criterion... Then we will show how our new method takes into account nonplanar surfaces in the hierarchical resolution process.
\end{abstract}

Key-words: Computer graphics, Global illumination, Hierarchical resolution, Progressive algorithm, BRDF, glossy reflection, Curved surfaces 


\section{Un Algorithme Progressif pour le Transport Trois Points}

\section{Résumé :}

Une formulation "trois points" de l'équation de rendu est nécessaire lorsque l'on souhaite calculer l'illumination globale d'un environnement comportant des surfaces dont la fonction de réflectance bidirectionnelle est quelconque. L'utilisation de la technique des éléments finis pour le calcul de la solution mène à un systeme d'équations linéaires dont la matrice est cubique, ce qui est coûteux en temps de calcul et mémoire. Une approche hiérarchique est nécessaire. Aupperle et al. ont proposé un algorithme hiérarchique trois points pour le calcul d'illumination globale en présence de réflexions rugueuses. Nous présentons dans cet article les améliorations fondamentales que nous apportons à une telle méthode : shooting, "lazy" push-pull, critère de subdivision photométrique... Puis nous exposons notre technique permettant de prendre en compte des surfaces non planes dans la résolution des calculs d'échanges.

Mots-clé : Synthèse d'images, Illumination globale, Résolution hiérarchique, Algorithme progressif, BRDF, Réflection rugueuse, Surfaces courbes 


\title{
A Progressive Algorithm For Three Point Transport

\author{
Reynald Dumont ${ }^{*+}$, Kadi Bouatouch ${ }^{+}$, Philippe Gosselin
} \\ * CRIL Ingénierie, 5 Sq. Chêne Germain, 35510 Cesson-Sévigné, France \\ ${ }^{+}$IRISA, Campus Universitaire de Beaulieu, 35042 Rennes Cedex, France
}

\begin{abstract}
When computing global illumination in environments made up of surfaces with general Bidirectional Reflection Distribution Function, a three point formulation of the rendering equation can be used. Brute-force algorithms can lead to a linear system of equations whose matrix is cubic, which is expensive in time and space. The hierarchical approach is more efficient. Aupperle et al. proposed a hierarchical three point algorithm to compute global illumination in presence of glossy reflection. We present in this paper some improvements we brought to this method: shooting, "lazy" push-pull, photometric subdivision criterion... Then we will show how our new method takes into account non-planar surfaces in the hierarchical resolution process.
\end{abstract}

\section{Introduction}

Radiosity and ray-tracing techniques make strong assumptions about the characteristics of the used materials. The first one assumes that all surfaces are perfectly diffuse and the second, totally view dependent, replaces the indirect diffuse component by an ambient term. A few years ago, some approaches, called two-pass methods, mixed both techniques to handle at once specular and lambertian surfaces ([ 15 ], [ 18 ] and [ 19 ]). The global diffuse and the global specular components are evaluated at the first and the second pass respectively.

Conversely, the one-pass methods compute in one pass the global diffuse and the global specular components. To compute an image with these methods a rendering step is needed. Two kinds of one-pass method have been devised in the past years to take account of glossy reflection.

The first one relies on the Monte Carlo method such as random walk. It is relatively easy to implement and can be used with complex geometry and general reflectance functions but is notoriously slow since too many rays have to be cast to get an accurate result ([ 7 ], [ 10 ], [ 11 ], [ 13 ], [ 20 ]).

The second technique makes use of wavelets ([ 5 ], [ 12 ], [ 14 ]) and spherical harmonics ([ 16 ]) to compute global illumination. Indeed, wavelets or spherical harmonics are used as basis functions to express the radiance of each patch for each direction. In [ 5 ], the object surfaces as well as the direction space are adaptively subdivided (two point method). In [ 12 ][ 14 ], the rendering equation is expressed in terms of three point transport and only the environment surfaces are meshed into patches. In case of constant basis functions, Aupperle and Hanrahan proposed an interesting specific method for three point transport which computes the radiance from a patch to another one. The data structures needed by the wavelet-based methods require an important memory capacity which limits them practically. Regarding the spherical harmonics-based methods, they need a high number of basis functions and make use of a uniform meshing of the object surfaces. Like the wavelet-based methods, Aupperle's method has the advantage to be hierarchical but still requires an important memory storage because of the huge number of links (between interactions) and a too fine meshing due to the non use of a photometry-based subdivision criterion.

One solution to overcome the memory limitation is to use the methods described by Teller and Airey ([ 17 ], [ 1 ]). These methods relie on a binary space subdivision of the environment into 3D cells. A polygon in a cell $\mathrm{C}$ sees only the polygons lying in $\mathrm{C}$ as well as those visible through the holes (also termed portal) in its boundary, like windows and doors for building interiors. To gather energy impinging on each patch within a cell $\mathrm{C}$, we need to store in memory only the cells $C_{i}$ visible from $C$, which limits the memory storage. In spite of the efficiency of this kind of method, the problem of memory storage still remains in a cell containing many surfaces and many glossy reflectors.

To overcome these difficulties we propose in this paper an improvement as well as an extension of Aupperle's method to non-planar subdivision.

The organization of this paper is as follows. Section 2 is an outline of our work. Section 3 briefly recalls Aupperle's et al. algorithm. Section 4 describes the different improvements we have brought to Aupperle's method such as: Shooting, photometric subdivision criterion, efficient Push/Pull and rendering. During the subdivision process, objects can be subdivided into four subsurfaces. Some difficulties arise when surfaces in the environment are not planar (i.e. the four subsurfaces resulting from the subdivision may not lie on the same plane). Section 5 gives one solution to this problem.

\section{Outline}

Our approach is based on Aupperles's work which relies on a one pass method as said previously. Recall that with this method, the number of links stil remains high and the meshing is too fine due to the non-use of a photometry based subdivision criterion. 
Once an iteration (shooting or gathering) has been completed, we propose to remove all the created links to save memory. Moreover, to avoid a too fine meshing, we make use of a photometry-based subdivision criterion. In our implementation we have opted for shooting rather than gathering because useful images can be produced very early in the calculation process. To improve the efficiency of our algorithm we propose an accelerated push-pull process called "lazy" push-pull from now on. Unlike Aupperle's method our algorithm is totally view independent, say it does not consider the eye as a small patch within the environment.

Another important contribution brought by our work is the handling of curved surfaces. We will see how a patch can be subdivided into four non coplanar sub-patches.

\section{Hierarchical Algorithm for Three Point Light Transport}

\subsection{The Radiance Equation}

\subsubsection{Three point transport}

Global illumination in a general environment may be expressed in terms of three point light transport between a triplet of surfaces A, A' and A", (i.e. flux emitted by A' towards A", when illuminated by A).

The radiance from a point $\mathrm{x}^{\prime}$ towards a point $\mathrm{x}$ " is defined as the emitted flux, per unit solid angle, per unit projected area, originating at $x^{\prime}$ in the direction of $x^{\prime}$ '.

We can therefore express the radiance $L\left(x^{\prime}, x^{\prime \prime}\right)$ at a point $\mathrm{x}^{\prime}$ towards a point $\mathrm{x}^{\prime \prime}$, when illuminated by a point $\mathrm{x}$ as:

$$
L\left(x^{\prime}, x^{\prime \prime}\right)=f r\left(x, x^{\prime}, x^{\prime \prime}\right) \cdot L\left(x, x^{\prime}\right) \cdot G\left(x, x^{\prime}\right) \cdot d x
$$

Equation 1

where the geometric term $\mathrm{G}$ is given by $G\left(x, x^{\prime}\right)=\frac{\cos \theta_{i} \cdot \cos \theta_{A}}{\left\|x-x^{\prime}\right\|^{2}} \cdot V\left(x, x^{\prime}\right)$ (See Figure 1$)$.

For simplicity we leave wavelength out of our equations. The term fr is called bidirectional reflectance distribution function or BRDF and V is a boolean used to express occlusions between $\mathrm{x}$ and $\mathrm{x}$ '.

By integrating over A, A' and A" we can easily prove that the total flux emitted by A' towards A" when illuminated by A is equal to:

$$
\Phi_{A A^{\prime} A^{\prime \prime}}=\int_{A} \int_{A^{\prime}} \int_{A^{\prime \prime}} f r\left(x, x^{\prime}, x^{\prime \prime}\right) \cdot L\left(x, x^{\prime}\right) \cdot G\left(x, x^{\prime}\right) \cdot G\left(x^{\prime}, x^{\prime \prime}\right) \cdot d x^{\prime \prime} d x^{\prime} d x
$$

Equation 2

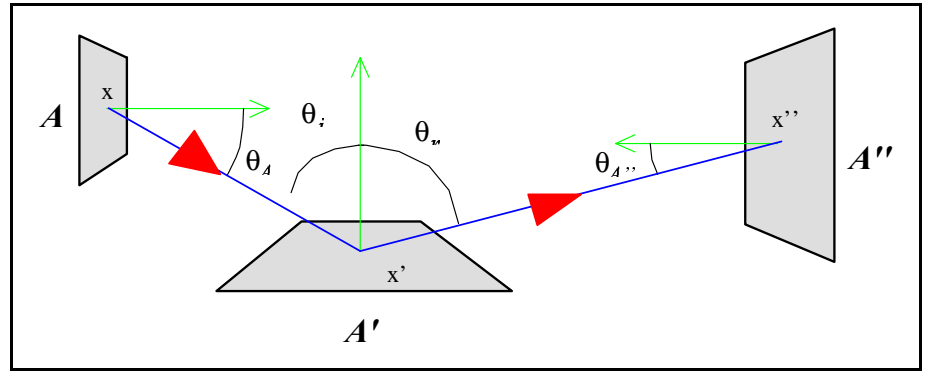

Figure 1: Geometry for three point transport

\subsubsection{Discrete form of Three Point Transport}

We can rewrite Equation 2 in a discrete form with surface elements $A_{i}, A_{j}$ and $A_{k}$ of surfaces $A, A^{\prime}$ and A" respectively, so as radiances, BRDF's and geometric terms are nearly constant over each surface element. Then we can obtain a discretized form of the radiance equation:

$$
L_{A_{j} A_{k}}=\sum_{i} L_{A_{i} A_{j}} R_{A_{k} A_{j} A_{i}}
$$

Equation 3

where $\mathrm{L}_{\mathrm{A}_{j} \mathrm{~A}_{\mathrm{k}}}$ (resp. $\mathrm{L}_{\mathrm{A}_{\mathrm{i}} \mathrm{A}_{\mathrm{j}}}$ ) is the radiance emitted by $\mathrm{A}_{\mathrm{j}}$ towards $\mathrm{A}_{\mathrm{k}}$ (resp. $\mathrm{A}_{\mathrm{i}}$ towards $\mathrm{A}_{\mathrm{j}}$ ).

Taking into account self-emission we thus have a formulation of the light transport between $A_{j}$ and $A_{k}$ when $A_{j}$ is illuminated by all the surface elements $A_{i}$ in the environment:

$$
L_{A_{j} A_{k}}=L_{A_{j} A_{k}}^{\varepsilon}+\sum_{i} L_{A_{i} A_{j}} R_{A_{k} A_{j} A_{i}}
$$




\subsubsection{Area Reflectance}

The quantity $R_{A_{k} A_{j} A_{i}}$ is a formulation of reflection over three surfaces $A_{i}, A_{j}, A_{k}$ and has a physical significance: it is no more than the proportion of energy originating at surface A and reflected by surface A' towards A':

$R_{A_{i} A_{j} A_{k}}=\frac{\int_{A_{i}} \int_{A_{j}} \int_{A_{k}} f r\left(x, x^{\prime}, x^{\prime \prime}\right) G\left(x, x^{\prime}\right) G\left(x^{\prime}, x^{\prime \prime}\right) d x^{\prime \prime} d x^{\prime} d x}{\int_{A_{i}} \int_{A_{j}} G\left(x, x^{\prime}\right) d x^{\prime} d x}=\frac{\text { Flux transported from } A_{i} \text { to } A_{j} \text { that is reflected towards } A_{k}}{\text { Flux transported from } A_{i} \text { to } A_{j}}$

This term is called area reflectance and satisfies both energy conservation and symmetry properties. With the assumption that the BRDF and geometric terms are relatively constant over each surface element, we can thus obtain a simplified expression:

$$
R_{A_{k} A_{j} A_{i}}=\pi \cdot F_{A_{j} A_{i}} \cdot f r_{A_{k} A_{j} A_{i}}
$$

Equation 5

The term $\mathrm{F}_{\mathrm{A}_{\mathrm{j}} \mathrm{A}_{\mathrm{i}}}$ represents the form factor between $\mathrm{A}_{\mathrm{j}}$ and $\mathrm{A}_{\mathrm{i}}$ and $f r_{A_{k} A_{j} A_{i}}$ is the discretized value of the BRDF over surfaces $\mathrm{A}_{\mathrm{k}}, \mathrm{A}_{\mathrm{j}}, \mathrm{A}_{\mathrm{i}}$. The accuracy of the estimates for $\mathrm{F}$ and $f r$ depends on the size of the patches over which reflectance is computed, relative to the distance between them. As the relative size decreases so do the computation errors leading then to the adaptive refinement strategy as shown in the following.

\subsection{The Algorithm}

\subsubsection{Principle}

Equation 4 leads to a linear system of equations whose solution can be obtained by gathering. The unknowns are the radiances $\mathrm{L}_{\mathrm{AiAj}}$ with $\mathrm{i}$ and $\mathrm{j}$ ranging from 1 to $\mathrm{n}, \mathrm{n}$ being the number of patches. The complexity of the system is $O\left(n^{3}\right)$ which makes the resolution problem untractable. That is why Aupperle et al. proposed a hierarchical algorithm to solve this system. While hierarchical radiosity operates on patches and refines the links between them, the three point transport algorithm operates on patch-to-patch interactions and refines the links between them. Figure 2 gives the data structure associated with an interaction. As refinement results, the interactions are subdivided under the form of hierarchy as shown in Figure 3. In this figure the contribution of the interaction IJ to the interaction JK is illustrated by four contribution links: $\mathrm{I}_{1} \mathrm{~J} \rightarrow \mathrm{JK}, \mathrm{I}_{2} \mathrm{~J} \rightarrow \mathrm{JK}, \mathrm{I}_{3} \mathrm{~J} \rightarrow \mathrm{JK}$ and $\mathrm{I}_{4} \mathrm{~J} \rightarrow \mathrm{JK}$ ).

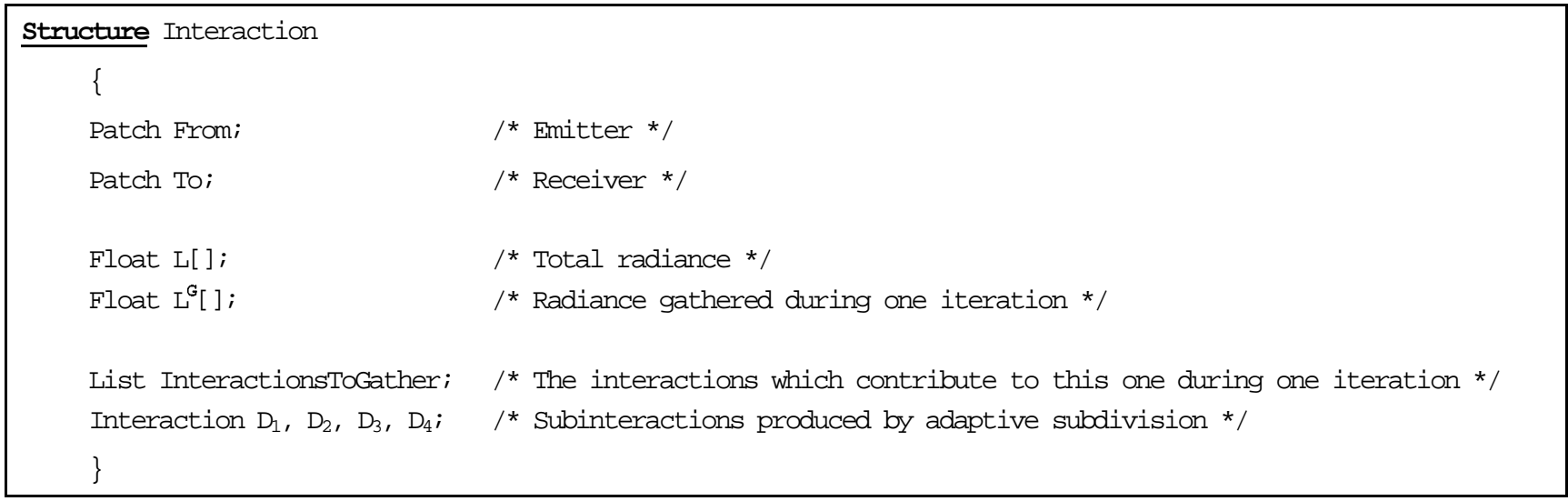

Figure 2: Interaction Data Structure

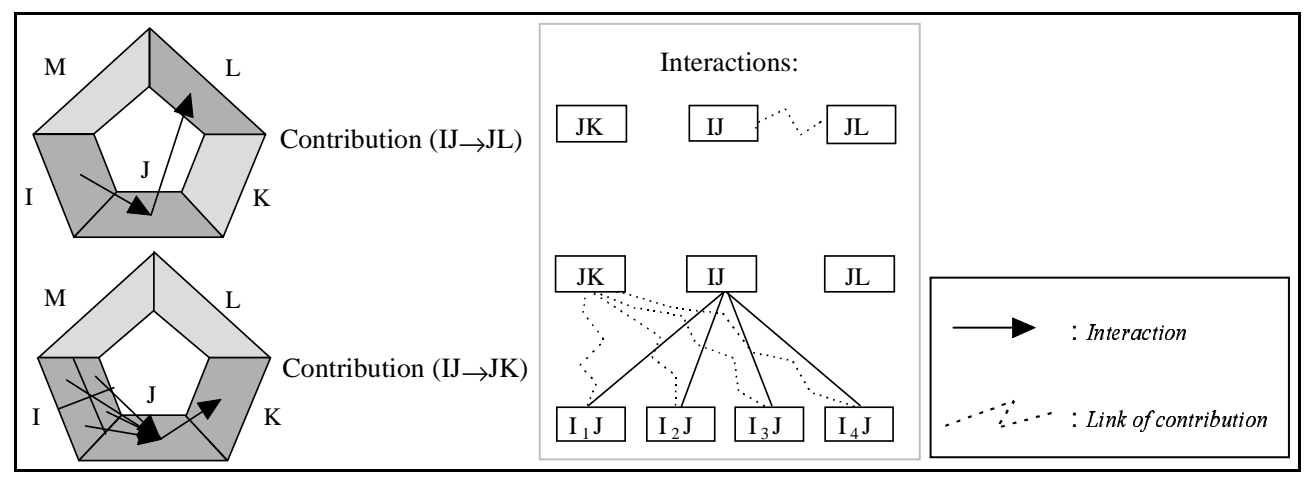

Figure 3: Hierarchical discrete three point transport 


\subsubsection{Refinement}

The refinement procedure RefineAndLink () computes pairs of interactions by subdividing and recursively refining links if the error estimates exceed specified error bounds, then links these interactions if these bounds are satisfied or no further subdivision is possible. FFErr and FrErr are the bounds for the geometric and the reflection errors respectively and MinArea is the minimum area for a patch (See Figure 4 and Figure 5).

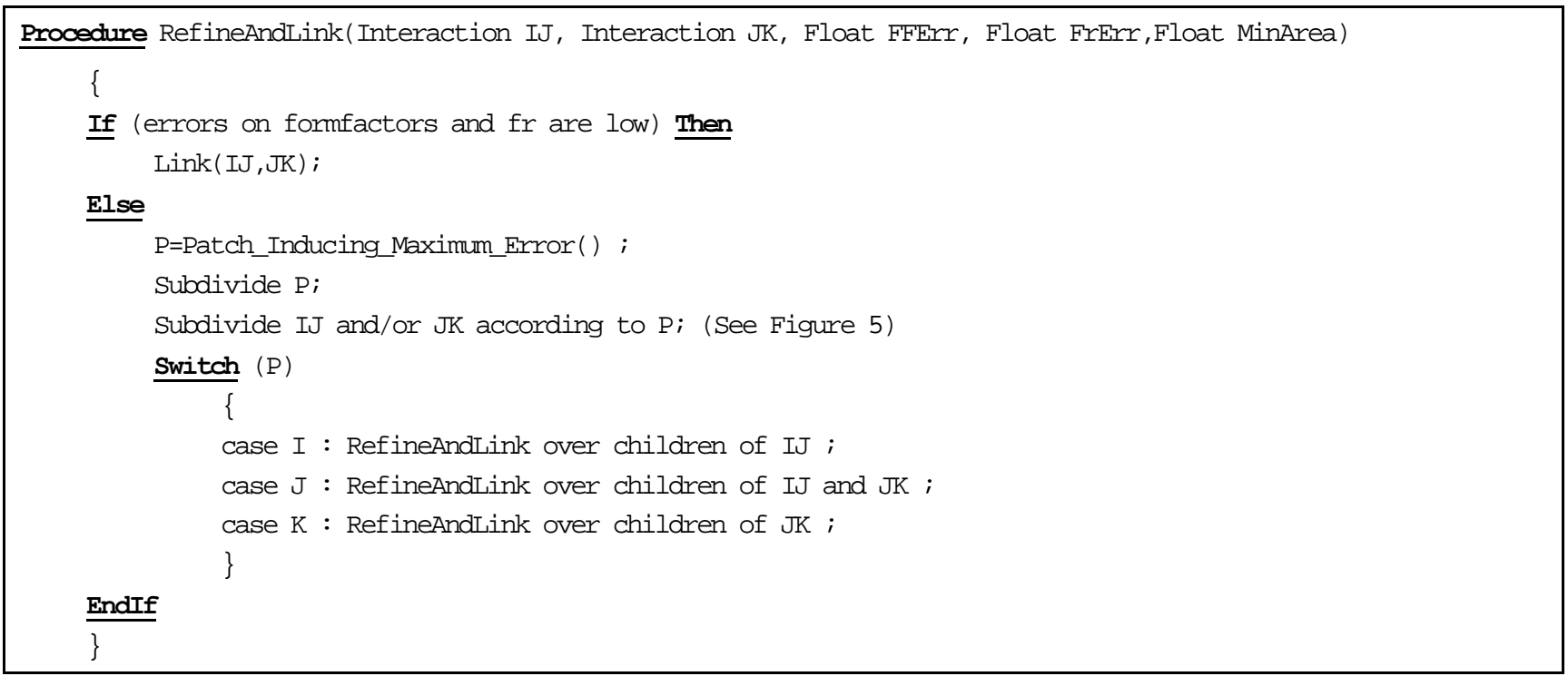

Figure 4: RefineAndLink() Procedure.

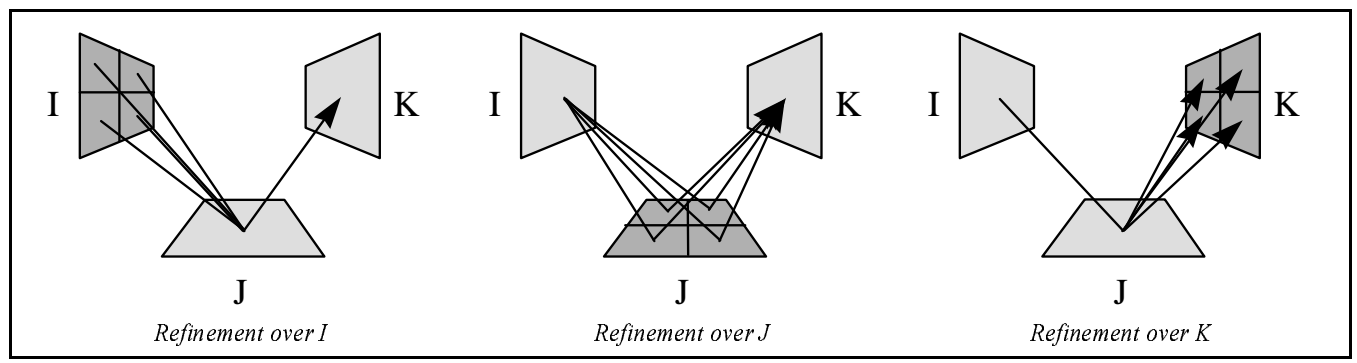

Figure 5: The three potential case of subdivision.

\subsubsection{Gathering Radiance}

Once the refinement process has been performed, each interaction gathers radiance from the interactions to which it is linked. This process continues till convergence (Figure 6).

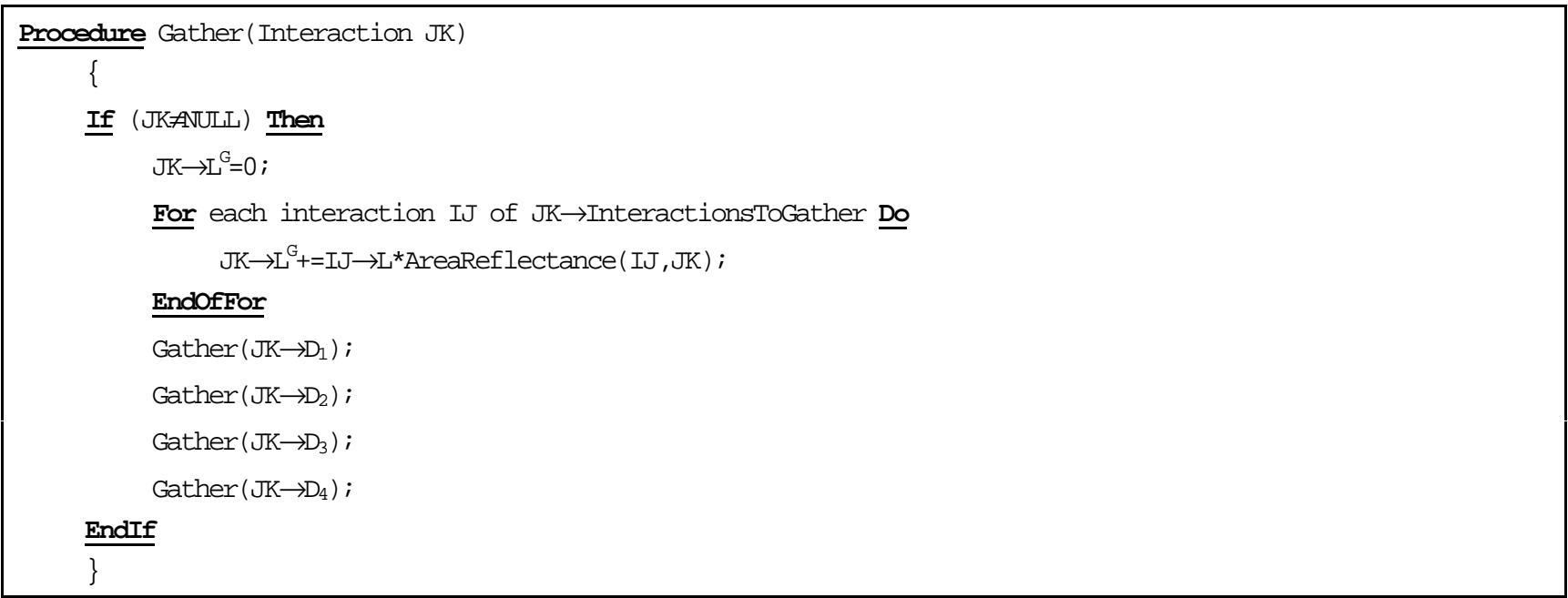

\section{Figure 6: Gathering() Procedure}

Note that the eye is considered as a small patch causing no occlusions nor reflections. 


\subsubsection{PushPull Radiance}

Recall that an interaction IJ between two input patches I and $\mathrm{J}$ is represented by a hierarchy of interactions between subpatches of I and J. For a reason of coherence, the gathered radiances must be distributed through each hierarchy. Indeed, a radiance is pushed down unchanged from a node to its children and area averaged when it is pulled from a node to its ancestors.

Given an interaction IJ, if patch I is subdivided, the pulled radiance is $L_{I J}=\sum_{m=1}^{4} \frac{A_{I_{m}}}{A_{I}} L_{I_{m} J}$, or if $\mathrm{J}$ is subdivided, it is equal to $L_{I J}=\sum_{m=1}^{4} \frac{A_{J_{m}}}{A_{J}} L_{I J_{m}}$.

\subsection{Discussion}

In spite of the advantages brought by the three point transport algorithm, some problems remain not solved. Indeed, firstly, since the used refinement criterion does not account for photometric quantities, the number of interactions still remains important, which requires a large memory size and an important computation time. Secondly, the algorithm is view dependent since the view point is considered as a small patch within the environment. And thirdly, only planar patches are considered.

\section{Our Approach}

\subsection{Principle}

As said before, Aupperle's method is a demanding process in terms of computing time and memory ressources, which limits the method practically. In this section we will describe in turn the different solutions to overcome these difficulties: Shooting, photometric subdivision criterion, efficient Push/Pull and rendering.

Unlike Aupperle's approach, we have opted for a shooting technique instead of gathering in order to get useful images at the earlier steps of the resolution and to reduce the memory size needed for storing the data structures thanks to a dynamic link management strategy. With this latter the spatial complexity is reduced to $O\left(n+k^{2}\right)$ (rather than $O\left(n+k^{3}\right)$ ), where $\mathrm{n}$ is the number of elements at the finest level of subdivision and $\mathrm{k}$ is the number of input patches. Figure 7 gives our algorithm.

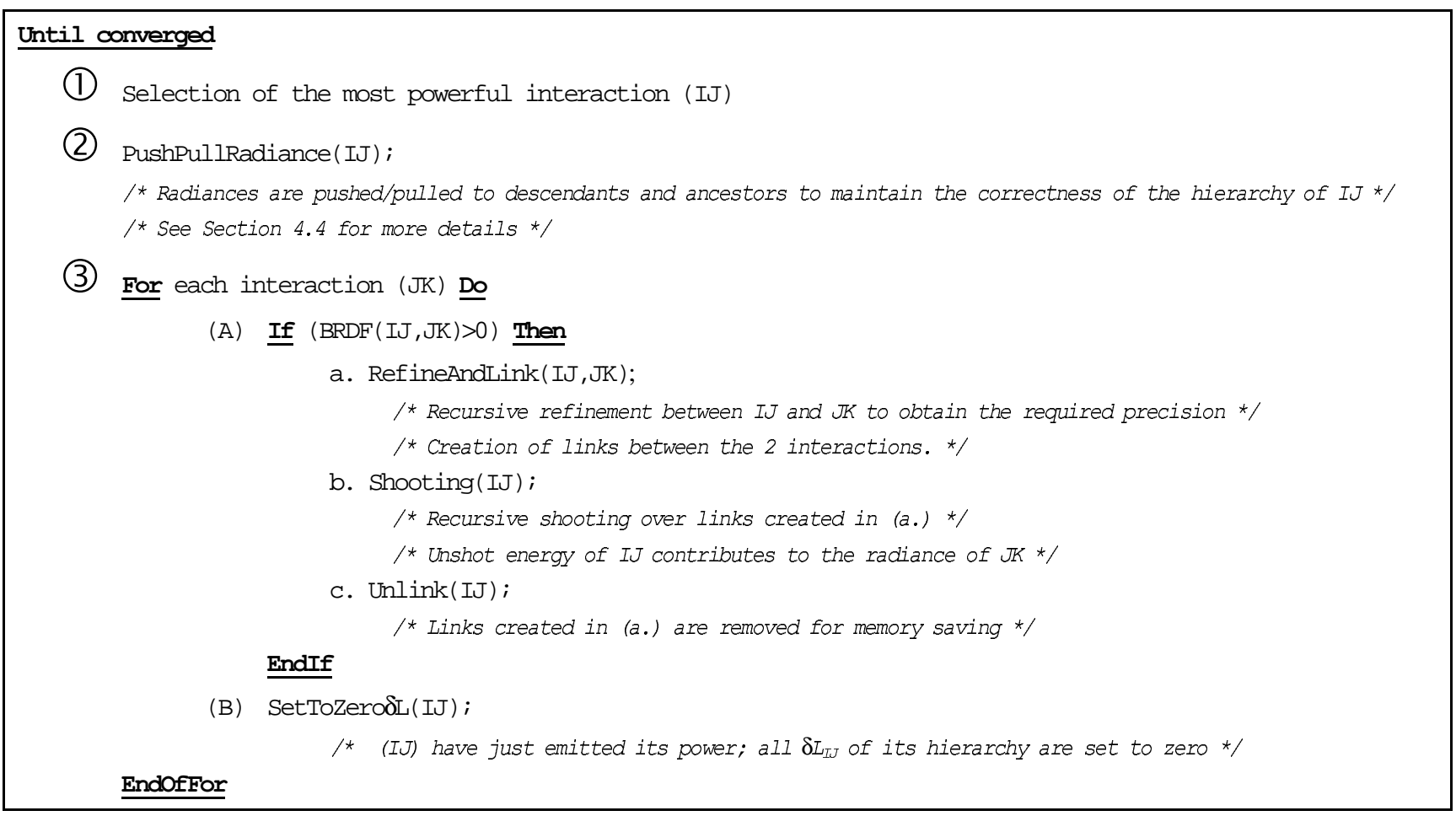

Figure 7: Main Algorithm

Rendering is performed by a gathering method combining ray-tracing and Monte Carlo method. 


\subsection{Shooting Radiance}

The gathering method proposed by Aupperle needs the storage of all the links between hierarchies of interaction. Since a too large amount of memory is required for this information, we have opted for a different approach based on shooting.

Our shooting algorithm allows a dynamic management of links and then only few links are always kept in memory. To achieve this, links between the emitting and the receiving interactions are first created. Once the shooting operation has been performed these links are deleted. This process allows to save memory at the expense of an extra computing time.

The data structure associated with an interaction becomes now:

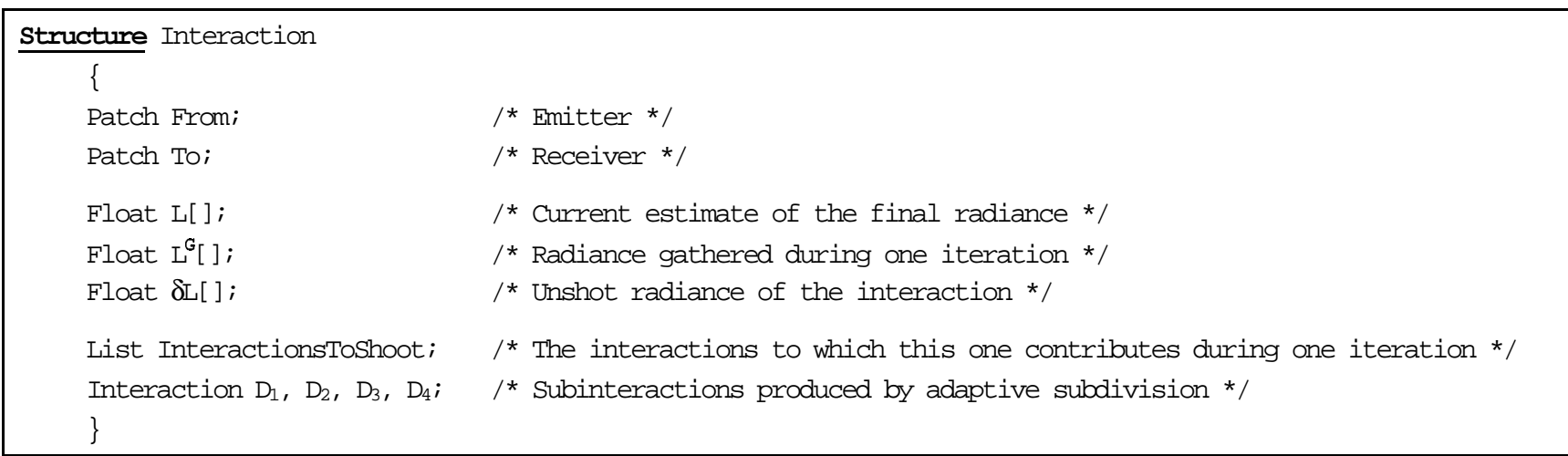

Figure 8: The New Data Structure

The computation of the shooting contribution consists of three steps:

1. Select the next shooting interaction IJ corresponding to the greatest unshot flux $\delta \phi$ :

$\delta \phi_{I J}=\pi \cdot A_{I} \cdot F_{I J} \cdot \delta L_{I J}$

2. Compute the contribution of interaction IJ to each other interaction JK:

$L_{J K}^{G}=\delta L_{I J} R_{K J I} \quad$ (for each node of IJ linked to JK)

3. Reset the unshot radiance:

$\delta L_{I J}=0 \quad$ (for each node of $\left.I J\right)$

Note that, to achieve this, this shooting procedure looks for all the existing links between the nodes belonging to the hierarchies associated with the emitting and the receiving interactions (Figure 9).

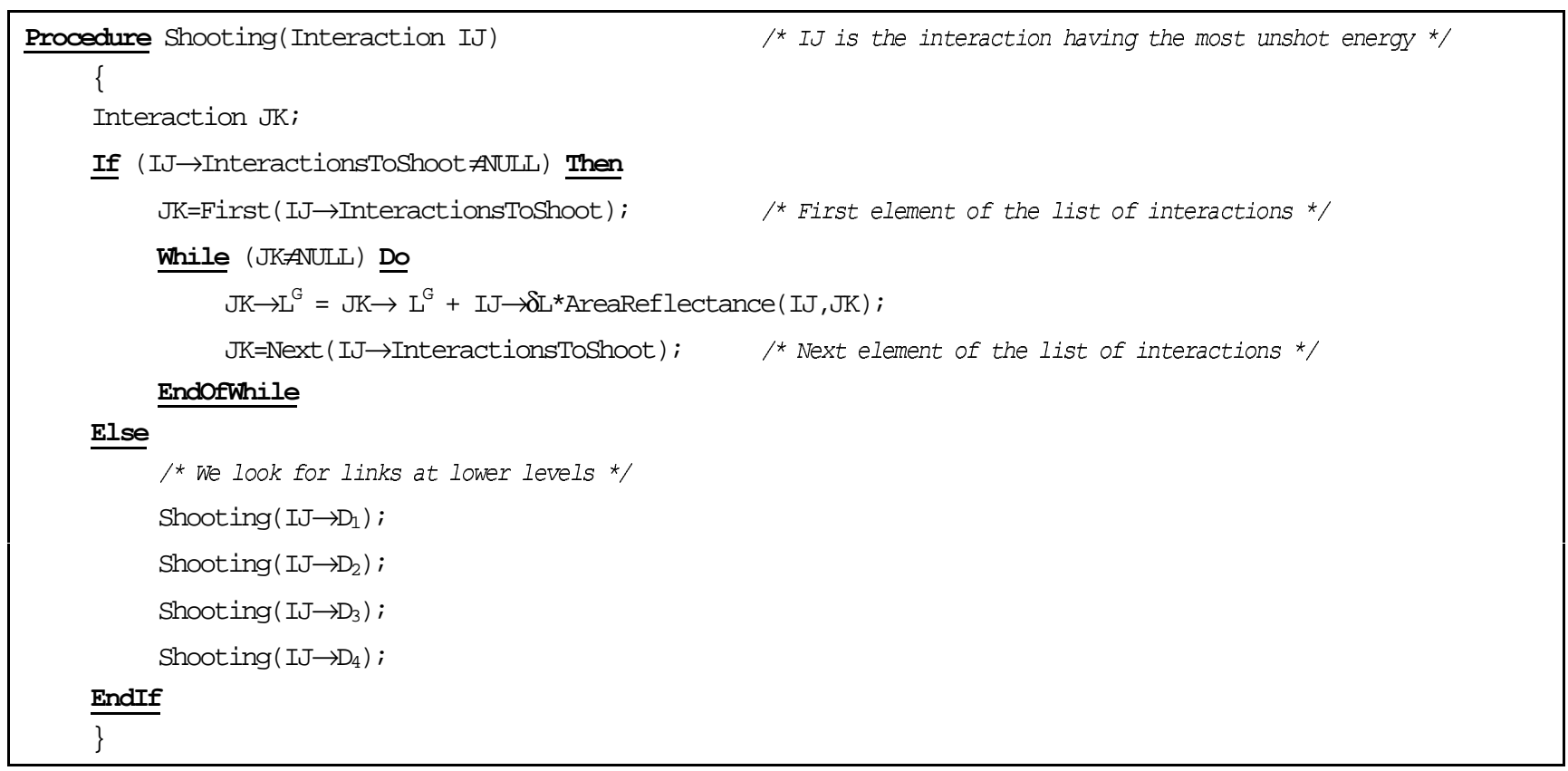

Figure 9: The Shooting Procedure 
(1) IJ is selected for shooting

(2)

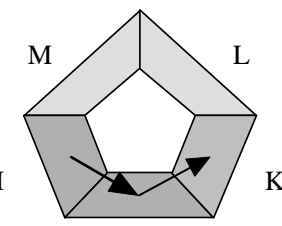

J

RefineAndLink(IJ,JK); Shoot(IJ);

Unlink(IJ):
I

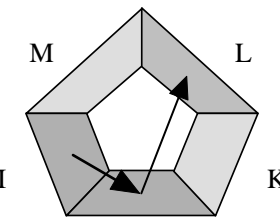

$\mathrm{J}$

RefineAndLink(IJ,JL); Shoot(IJ);

Unlink(IJ):

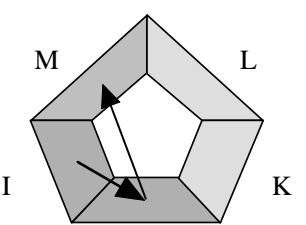

$\mathrm{J}$

RefineAndLink(IJ,JM);

Shoot(IJ);

Unlink(IJ):

(3) $\delta \mathrm{L}_{\mathrm{IJ}}=0$

Figure 10: One iteration

\subsection{Photometric Subdivision Criterion}

Like for hierarchical radiosity (with BF criterion as in [ 8 ]), our subdivision criterion is based on the shot flux. In the RefineAndLink procedure the subdivision criterion becomes now:

If ( $\left(\phi_{\text {IJK }}\right.$ is low) or (errors on formfactors and fr are low)) Then

Link $(I J, J K)$;

Where $\phi_{\mathrm{IJK}}$ is the unshot flux which will be emitted by the interaction IJ towards the interaction JK.

\subsection{Efficient PushPull}

As the push/pull operation is time consuming, it is not necessary to perform it each time an interaction gets energy from the other interactions. The energy of an interaction is pushed/pulled only when it is chosen for shooting. However, to select the interaction having the greatest unshot energy, the radiance of each interaction between two input patches must be known. To this end, a variable $\delta \mathrm{L}^{\text {Root }}$ is associated with the root node of each hierarchy. Note that whenever an interaction of a hierarchy $H$ gets energy from the others, the variable $\delta \mathrm{L}^{\text {Root }}$ associated with $H$ is updated.

Let $\mathrm{IJ}$ be a receiving interaction, $\mathrm{I}_{\mathrm{m}}$ a subpatch of $\mathrm{I}$ and $\mathrm{J}_{\mathrm{n}}$ a subpatch of $\mathrm{J}$. It is easy to see that:

$$
\delta L^{\text {Root }}=\delta L^{\text {Root }}+L_{I_{m} J_{n}}^{G} \cdot \frac{\operatorname{AreaOf}\left(I_{m}\right)}{\operatorname{AreaOf}(I)} \cdot \frac{\operatorname{AreaOf}\left(J_{n}\right)}{\operatorname{AreaOf}(J)}
$$

Equation 6

\subsection{Final Gathering}

Recall that, unlike Aupperle's method, our approach is view independent. To compute an image of the environment as seen by an observer, a rendering step combines ray-tracing and the Monte Carlo method. Note that our approach is not a two pass method like in [15 ], [ 18 ] and [ 19].

Let's recall the radiance emitted at point $\mathrm{x}$ in direction $\vec{\omega}_{r}$ for an incidence $\vec{\omega}_{i}$ :

$$
L^{\text {out }}\left(x, \vec{\omega}_{r}\right)=\int_{2 \pi} \operatorname{fr}\left(\vec{\omega}_{i}, \vec{\omega}_{r}\right) \cdot L^{i n}\left(x, \vec{\omega}_{i}\right) \cdot \cos \theta_{i} \cdot d \omega_{i}
$$

This integral can be estimated with the Monte Carlo method by:

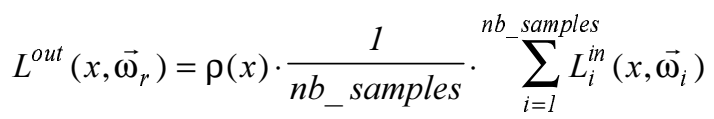

where

$$
\rho(\mathrm{x})=\int_{2 \pi} \operatorname{fr}\left(\vec{\omega}_{\mathrm{i}}, \vec{\omega}_{\mathrm{r}}\right) \cdot \cos \theta_{\mathrm{i}} \cdot \mathrm{d} \omega_{\mathrm{i}} \text { is the }
$$

reflectivity of the considered material at point $\mathrm{x}$.

To carry out the Monte Carlo method, a ray originating at the viewpoint is traced through each pixel. This ray intersects the scene at point $X_{S}$. From $X_{S}$, a number $n$ of secondary rays are traced according to the pdf $\operatorname{fr}\left(\vec{\omega}_{i}, \vec{\omega}_{r}\right) \cdot \cos \theta_{i} / \rho(x)(\operatorname{See}$ Figure 11a). Suppose that a secondary ray intersects the scene at point $X_{D}$ (See Figure 11b). The points $X_{S}$ and $X_{D}$ belong to two input patches $\mathrm{A}$ and $\mathrm{B}$ respectively. The problem now is to find the radiance at $\mathrm{X}_{\mathrm{D}}$ in direction of $\mathrm{X}_{\mathrm{S}}$. To 
achieve this, the hierarchy of interaction $\mathrm{AB}$ is traversed from the root to the leaf corresponding to the smallest subpatches containing the points $\mathrm{X}_{\mathrm{S}}$ and $\mathrm{X}_{\mathrm{D}}$ (See Figure 11c).

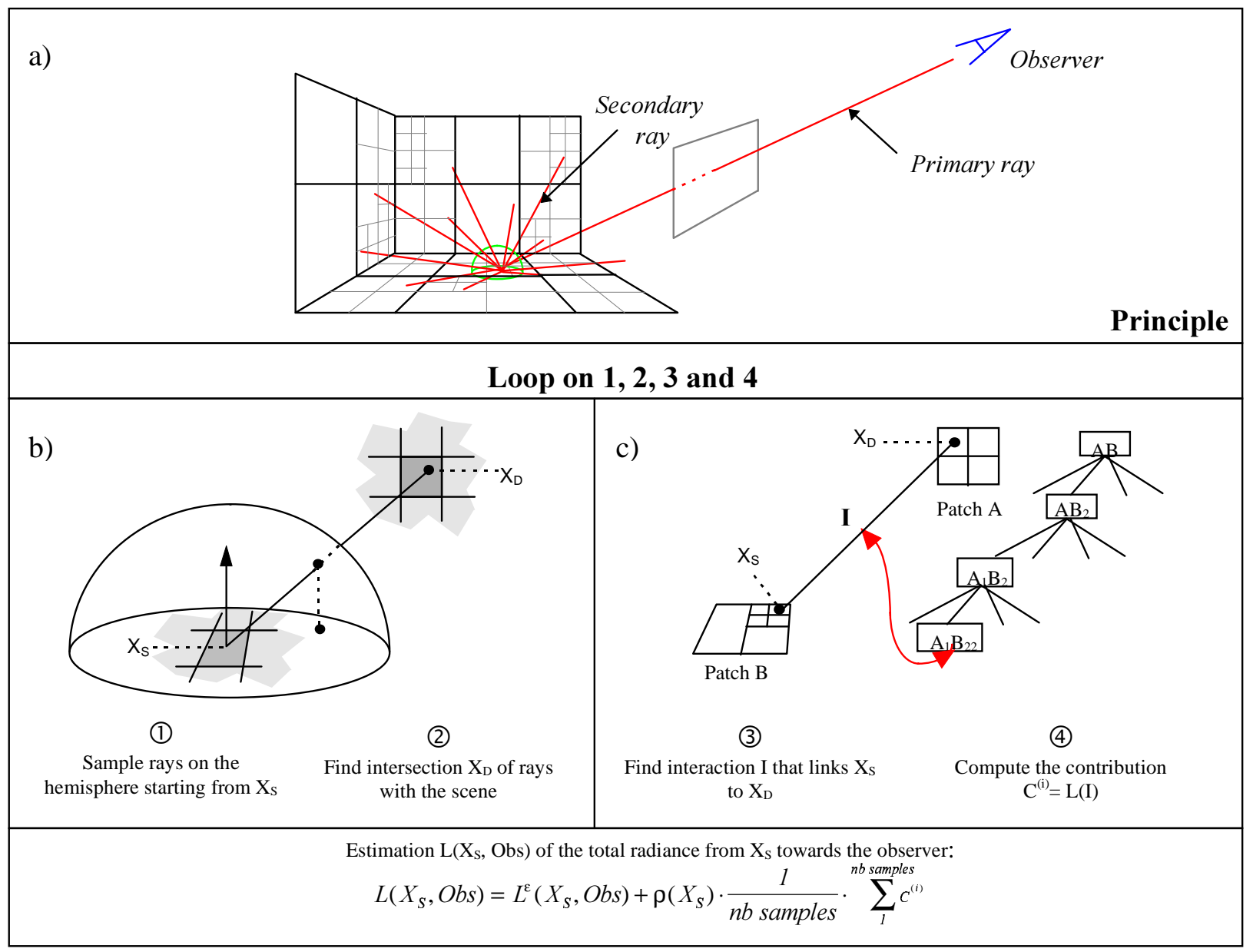

Figure 11: Final Gathering

\subsection{Visibility}

The method used for visibility calculation was deliberately left out of this paper since we use exactely the one given in [ 4 ]. In order to avoid computing it repeatedly, we store it in each interaction, under the form of a form factor multiplied by a visibility term.

\section{Non-Planar Subdivision}

\subsection{Motivation}

Let $\mathrm{S}$ be a curved surface within an environment for which global illumination computation has to be performed with our hierarchical algorithm. Thus, $\mathrm{S}$ must be initially approximated by a collection of polygons fitting the surface $\mathrm{S}$ (input patches). Let us call this approximation : "initial meshing".

Suppose that during the refinement process, an input patch of $\mathrm{S}$ is subdivided into 4 coplanar subpatches. If this curved surface has important curvatures then its initial meshing must be fine to closely approximate the surface $\mathrm{S}$. The consequence of this is the increase of the number of initial interactions which affects the performance of our hierarchical algorithm. To overcome this problem, we enable each input patch (and derived subpatches) to be subdivided into 4 noncoplanar subpatches in order to fit at best the curved surface. With this approach the initial meshing may be coarse, which reduces drastically the number of initial interactions, the number of links, and consequently the computation time as well as the required memory capacity.

In the following, interactions between non-coplanar subpatches of the same patch will be called secondary interactions while the others will be named primary (See Figure 12). 


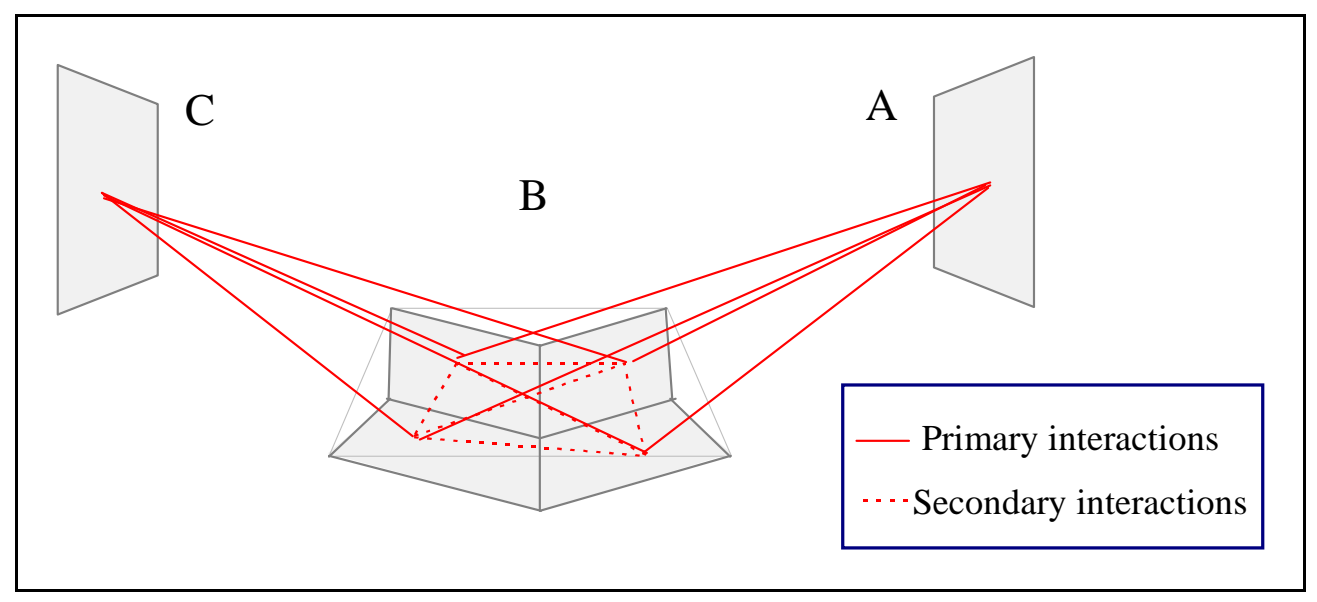

Figure 12 : Non-Planar subdivision

\subsection{Outline of the method}

It is obvious that the huge number of secondary interactions limits the hierarchical method practically. For this reason, we propose an extended hierarchical algorithm aiming at reducing these interactions as well as the associated links.

Let $\mathrm{A}$ and $\mathrm{C}$ be any two patches, and $\mathrm{B}$ an input patch of a curved surface. Suppose that the refinement process requires the subdivision of $\mathrm{B}$ into four non-coplanar subpatches $\mathrm{B}_{1}, \mathrm{~B}_{2}, \mathrm{~B}_{3}, \mathrm{~B}_{4}$. In our method, the energy transfer from $\mathrm{A}$ to $\mathrm{C}$ through $B$ is performed directly through the links $\left(A_{i} \rightarrow B_{i} C\right)$ and indirectly through only the links $\left(A_{i} \rightarrow B_{i} B_{j} \rightarrow B_{j} C\right)$. The other transfers like $\mathrm{AB}_{\mathrm{i}} \rightarrow \mathrm{B}_{\mathrm{i}} \mathrm{B}_{\mathrm{j}} \rightarrow \ldots \rightarrow \mathrm{B}_{1} \mathrm{~B}_{\mathrm{m}} \rightarrow \mathrm{B}_{\mathrm{m}} \mathrm{C}$ are ignored by our method to make the problem tractable. Note that $\mathrm{B}_{\mathrm{i}} \mathrm{B}_{\mathrm{j}}$ are secondary interactions which are not necessarily saved in memory (See Figure 13a).

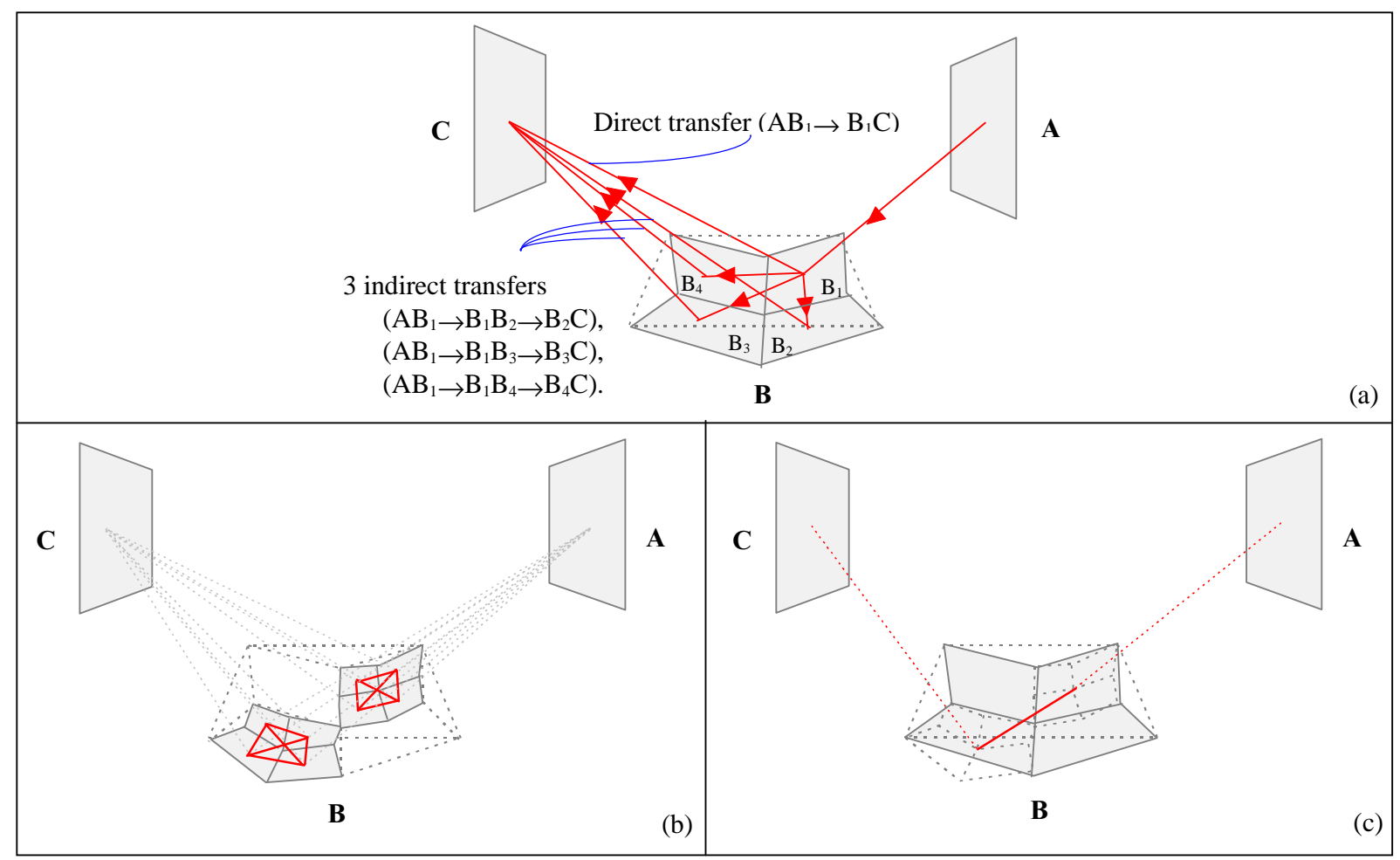

Figure 13 : Extended three point transport

Suppose that the patches $\mathrm{B}_{1}$ and $\mathrm{B}_{3}$ have to be subdivided in their turn into four subpatches $\mathrm{B}_{11}, \mathrm{~B}_{12}, \mathrm{~B}_{13}, \mathrm{~B}_{14}$ and $\mathrm{B}_{31}$, $\mathrm{B}_{32}, \mathrm{~B}_{33}, \mathrm{~B}_{34}$ respectively (See Figure $13 \mathrm{~b}$ ).

The energy transfer between $A$ and $C$ through $B_{1}$ is performed with the help of the direct links $\left(A_{1 i} \rightarrow B_{1 i} C\right)$ and of the indirect links $\left(\mathrm{AB}_{1 \mathrm{i}} \rightarrow \mathrm{B}_{1 \mathrm{i}} \mathrm{B}_{1 \mathrm{j}} \rightarrow \mathrm{B}_{1 \mathrm{j}} \mathrm{C}\right)$. The energy transfer between $\mathrm{A}$ and $\mathrm{C}$ through $\mathrm{B}_{3}$ is performed similarly.

Note that the secondary interactions like $\mathrm{B}_{1 \mathrm{i}} \mathrm{B}_{3 \mathrm{j}}$ are not accounted for to make our method tractable. However, their contributions are considered at a higher level, say when computing the contribution of the secondary interaction $\mathrm{B}_{1} \mathrm{~B}_{3}$ (See Figure 13c). 


\subsection{Data Structures}

Before giving details on the used data structure representing secondary interactions, let us recall that the number of secondary interactions entailed by the subdivision of a patch (belonging to a curved surface) is equal to sixteen (See Figure 14): $\mathrm{B}_{\mathrm{i}} \mathrm{B}_{\mathrm{j}}$, i and $\mathrm{j}$ ranging from 1 to 4 .

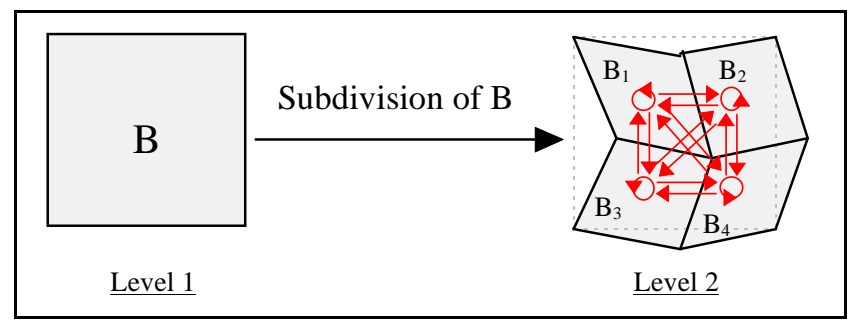

Figure 14 : Number of secondary interactions

The data structure associated with these interactions is a particular 16-Tree as shown in Figure 17. Indeed, as mentioned in the previous subsection, only the nodes $\mathrm{B}_{\mathrm{i}} \mathrm{B}_{\mathrm{i}}$ (at subdivision level one), $\mathrm{B}_{\mathrm{ij}} \mathrm{B}_{\mathrm{ij}}$ (at subdivision level two) and so on, have descendants.

\subsection{Refinement}

The new refinement algorithm is the following:

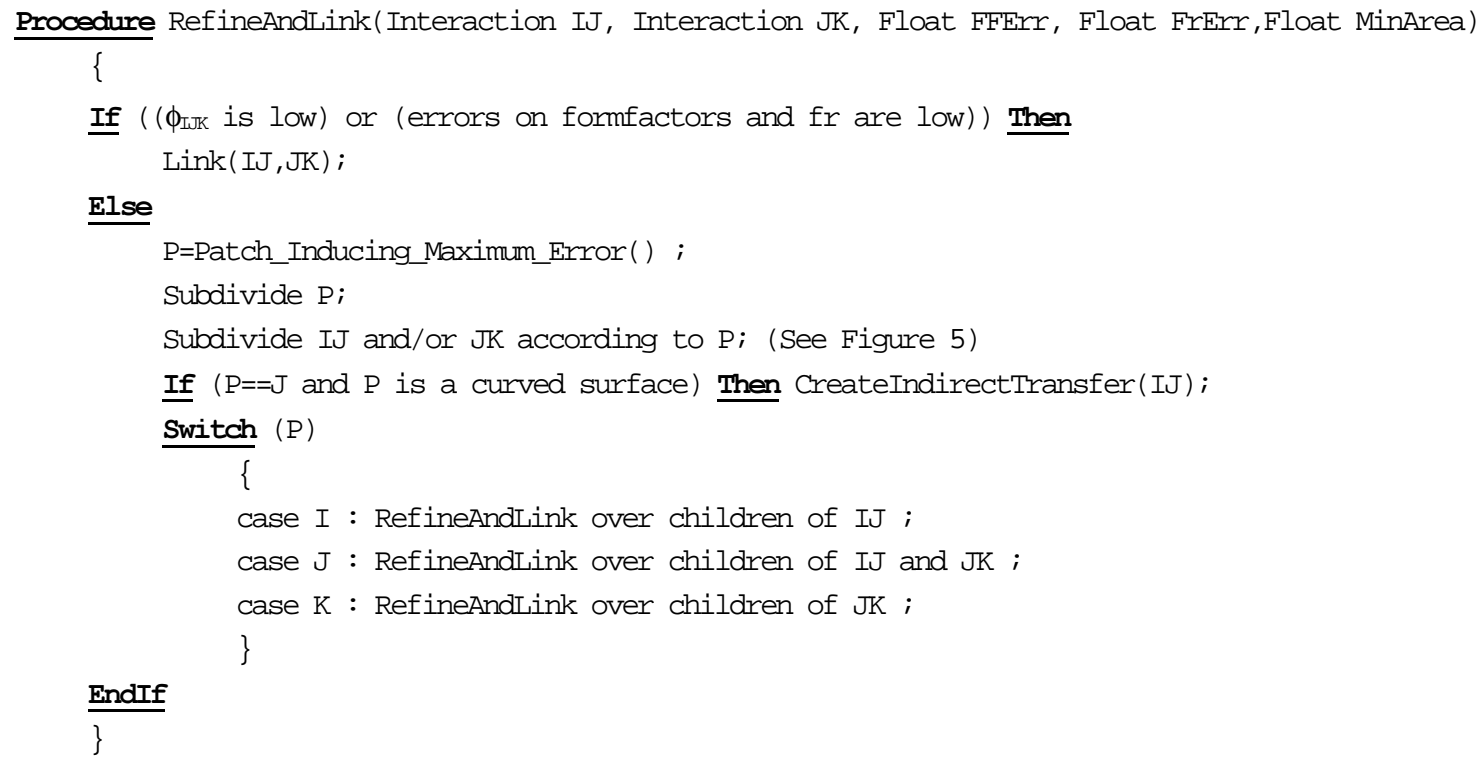

Figure 15 : Refinement with extended three point transport

CreateIndirectTransfer() is the procedure which is in charge of creating the secondary interactions and the associated links (Figure 16).

CreateIndirectTransfer (IJ)

\{

Let $I J$ be the initial primary interaction and JK the final primary interaction.

Let $J_{1}, J_{2}, J_{3}$ and $J_{4}$ be the sub-patches of $J$.

For each $I J_{\mathrm{n}}$ Do

For each $\mathrm{J}_{\mathrm{m}}$ with $m \neq n$ Do

/* Creation of the indirect transfer */

Link $I J_{n}$ to $J_{n} J_{m}$.

Link $J_{n} J_{m}$ to $J_{m} K$.

\section{EndofFor}

\section{EndOfFor}

\} 


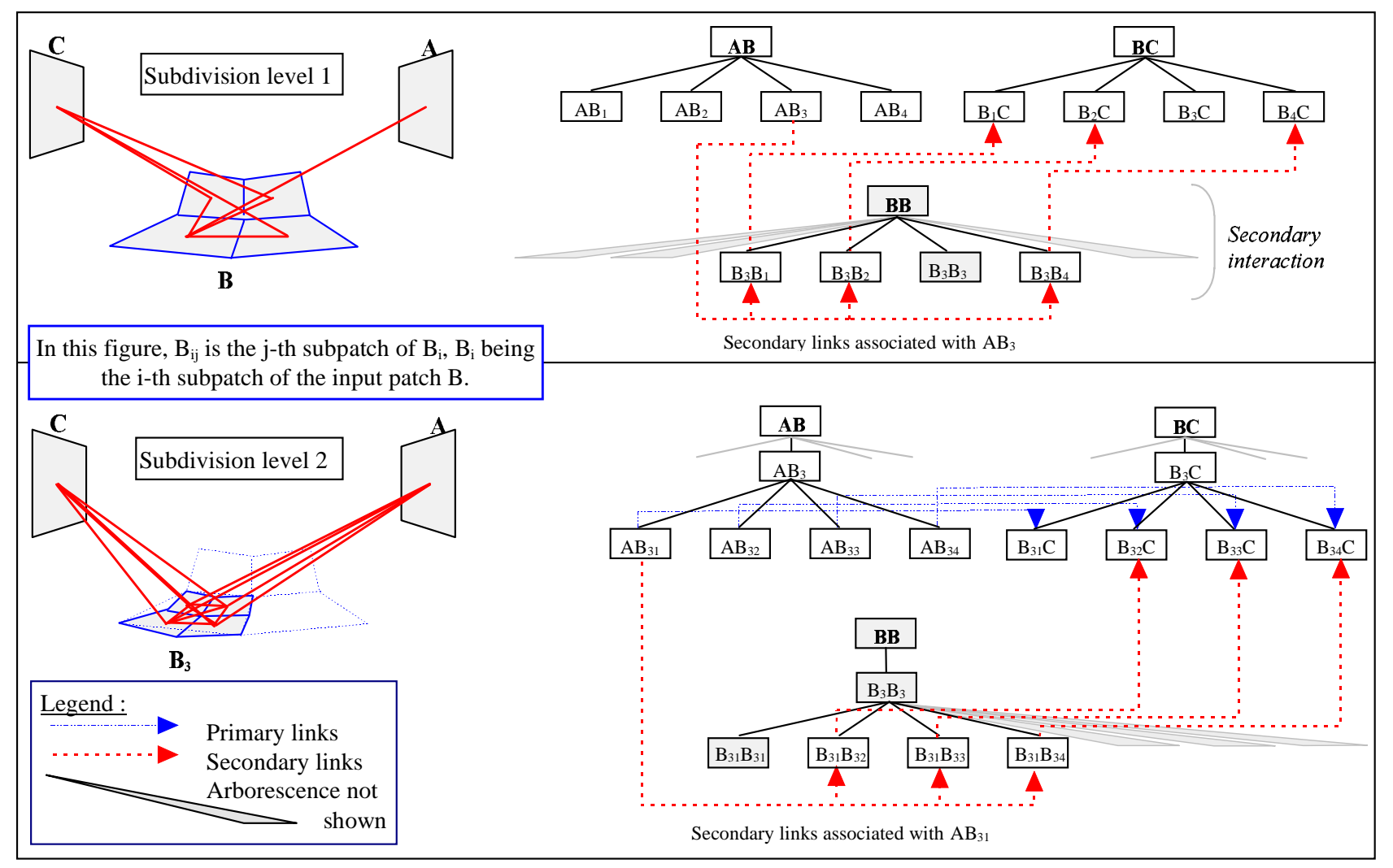

Figure 17 : Links between primary and secondary interactions after refinement.

\subsection{Indirect Shooting}

In case of secondary interactions the shooting algorithm is different from the one given in Figure 9.

To explain how the new shooting algorithm operates, let us consider Figure 18a. In this figure there are four patches A, $\mathrm{B}, \mathrm{C}$ and $\mathrm{D}, \mathrm{B}$ being a patch of a curved surface. The objective is to compute the energy transfers $\mathrm{AB} \rightarrow \mathrm{BC}$ and $\mathrm{AB} \rightarrow \mathrm{BD}$, through the direct links (Figure 18b) and the indirect links due to $\mathrm{B}$.

This amounts to compute the following indirect transfers: $A B_{i} \rightarrow B_{i} B_{j} \rightarrow B_{j} C$ and $A B_{i} \rightarrow B_{i} B_{j} \rightarrow B_{j} D$. We can remark that these two transfers have in common the transfers $A B_{i} \rightarrow B_{i} B_{j}$. This is why, for a reason of efficiency, we split the indirect shooting process into two steps: $A_{i} \rightarrow B_{i} B_{j}$ (Figure 18c) and $B_{i} B_{j} \rightarrow B_{j} C\left(\right.$ resp. $\left.B_{i} B_{j} \rightarrow B_{j} D\right)$ (Figure 18d).

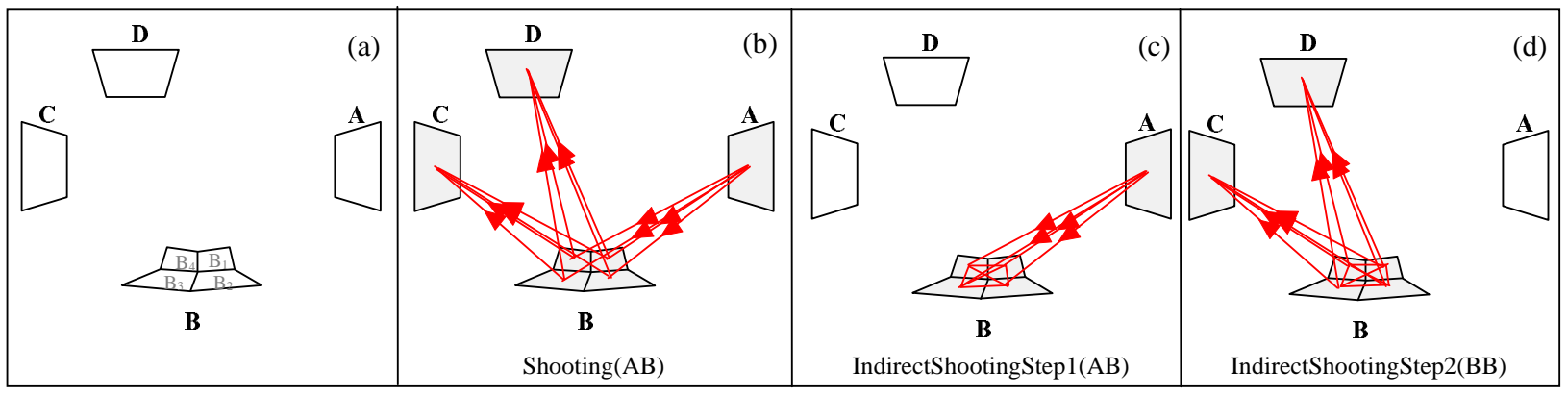

Figure 18 : Indirect shooting

The modified shooting is the following:

Shooting (IJ) ;

IndirectShootingStep1 (IJ) ;

IndirectShootingStep2 (JJ) ;

Figure 19: Shooting in presence of curved surfaces 


\subsection{Push/Pull}

The pushing operation is the same as for planar subdivision, while the pulling is slightly different. Indeed, unlike Aupperle's method (Section 3.2.4), the form factor terms must appear in the expression of the pulled radiances so as to account for the orientations of the non-coplanar subpatches belonging to the same patch. In other words, the pulled radiances are given by:

$$
L_{I J}=\sum_{k=1}^{4} \frac{A_{I_{k}} \cdot F_{J I_{k}}}{A_{I} \cdot F_{J I}} L_{I_{k} J} \text { or } L_{I J}=\sum_{k=1}^{4} \frac{A_{J_{k}} \cdot F_{J_{k} I}}{A_{J} \cdot F_{J I}} L_{I J_{k}} \text {, depending on the subdivided patch, where IJ is a node of }
$$

an interaction hierarchy and $\mathrm{I}_{\mathrm{k}} \mathrm{J}$ (or $\mathrm{IJ}_{\mathrm{k}}$ ) are its descendants.

\section{Results and Discussion}

Our algorithm has been implemented and tested with three kinds of scenes and with a Cook's and Torrance's reflection model. Each photometric quantity, like flux and radiance, is defined for ten wavelengths (spectral approach). The rendering process computes for each pixel a spectral radiance, projects this latter into the XYZ color space, and finally converts the resulting components into RGB components.

Scene 1 (see Figure 21) is a room containing a specular cube. The front facing wall is glossy while the other walls as well as the ceiling and the floor are diffuse. Scene 2 (see Figure 20) is a glossy curved surface illuminated by a small light source placed above it. Scene 3 is a more complex scene containing a curved surface (curtain) and planar surfaces (see Figure 22). Figure 23 and Figure 24 correspond to images of scene 3 for different view parameters. Figure 23 shows the curved shape of the curtain while Figure 24 put an emphasis on glossy reflection effects. Note that there are no pure specular reflections but only diffuse and glossy reflections.

The characteristics of these scenes are summarized in Table 1. We call potential elements the elements resulting from the uniform subdivision of each input patch at the finest resolution. Such a fine meshing induces a set of interactions (between its elements) which will be called potential interactions.

Table 2 gives for each scene the number of interactions and links. Recall that our shooting algorithm maintains in memory only the links created at the current iteration. We can remark (Table 2) that the ratio of the maximum number of these links to the total number of created links (obtained after convergence) is equal to $5.77 \%, 0.84 \%, 6.44 \%$ for scene 1 , 2 and 3 respectively. In addition, the number of interactions created by our hierarchical algorithm is far smaller than the number of potential interactions, even when a high precision is required (scene 2). To sum up, the results given by Table 2 demonstrates the efficiency of our algorithm in terms of memory saving.

Table 3 provides some results in terms of computing time. Before discussing these results let us define the convergence ratio $(\mathrm{CR})$ as: $C R=\frac{I F-R F}{I F}$, where the initial total flux IF is the sum of the light powers emitted by all the light sources and RF the total residual flux after convergence. Even with a high convergence ratio (95\%) the resolution times remain reasonable, which proves that our hierarchical algorithm is efficient.

To check the validity of the assumptions made by our algorithm when handling non-planar subdivisions, we have computed two images of scene 2 (with the same view parameters). The first one (Figure 20) has been computed by considering a coarse initial meshing (65 input patches) and non-planar subdivision, while the second has been generated with a very fine initial meshing (1025 input patches) and planar subdivision. The mean squared differences between the two normalized images for each component R, G, B and for the luminance Y are given in Table 4. We can see that these differences are insignificant even though the resolution process took $132 \mathrm{~s}$ for the first image and many hours for the second on a silicon graphics INDY R4600 (175 MHz).

\begin{tabular}{|c|c|c|c|}
\cline { 2 - 4 } \multicolumn{1}{c|}{} & Number of input patches & $\begin{array}{c}\text { Number of potential } \\
\text { elements }\end{array}$ & Number of light sources \\
\hline Scene 1 & 11 & 2816 & 1 \\
\hline Scene 2 & 65 & 4160 & 1 \\
\hline Scene 3 & 243 & 15552 & 7 \\
\hline
\end{tabular}

Table 1

\begin{tabular}{|c|c|c|c|c|}
\cline { 2 - 5 } \multicolumn{1}{c|}{} & $\begin{array}{c}\text { Number of } \\
\text { interactions after } \\
\text { convergence }\end{array}$ & $\begin{array}{c}\text { Number of potential } \\
\text { interactions }\end{array}$ & $\begin{array}{c}\text { Maximum number } \\
\text { of links stored in } \\
\text { memory }\end{array}$ & $\begin{array}{c}\text { Total number of } \\
\text { links created after } \\
\text { convergence }\end{array}$ \\
\hline Scene 1 & 922 & 7932672 & 118 & 2044 \\
\hline Scene 2 & 146764 & 17305600 & 2791 & 160553 \\
\hline Scene 3 & 107028 & 241849100 & 29000 & 450000 \\
\hline
\end{tabular}

Table 2 


\begin{tabular}{|c|c|c|c|c|}
\cline { 2 - 5 } \multicolumn{1}{c|}{} & Resolution time & $\begin{array}{c}\text { Rendering time } \\
\text { (image 256 256) }\end{array}$ & Convergence ratio & $\begin{array}{c}\text { Number of shooting } \\
\text { iterations }\end{array}$ \\
\hline Scene 1 & $63 \mathrm{~s}$ & $407 \mathrm{~s}$ & $95.11 \%$ & 50 \\
\hline Scene 2 & $132 \mathrm{~s}$ & $448 \mathrm{~s}$ & $94.70 \%$ & 100 \\
\hline Scene 3 & $758 \mathrm{~s}$ & $780 \mathrm{~s}$ & $90.20 \%$ & 150 \\
\hline
\end{tabular}

Table 3

\begin{tabular}{|l|c|c|c|c|}
\cline { 2 - 5 } \multicolumn{1}{c|}{} & $\mathbf{R}$ & $\mathbf{G}$ & $\mathbf{B}$ & $\mathbf{Y}$ \\
\hline Mean squared difference & 0.032776 & 0.009426 & 0.001416 & 0.016500 \\
\hline
\end{tabular}

Table 4

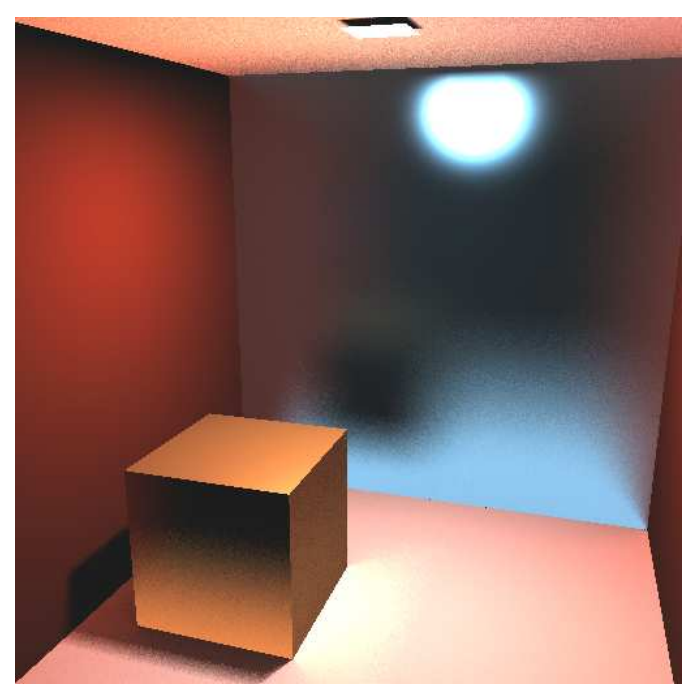

Figure 21: Scene 1

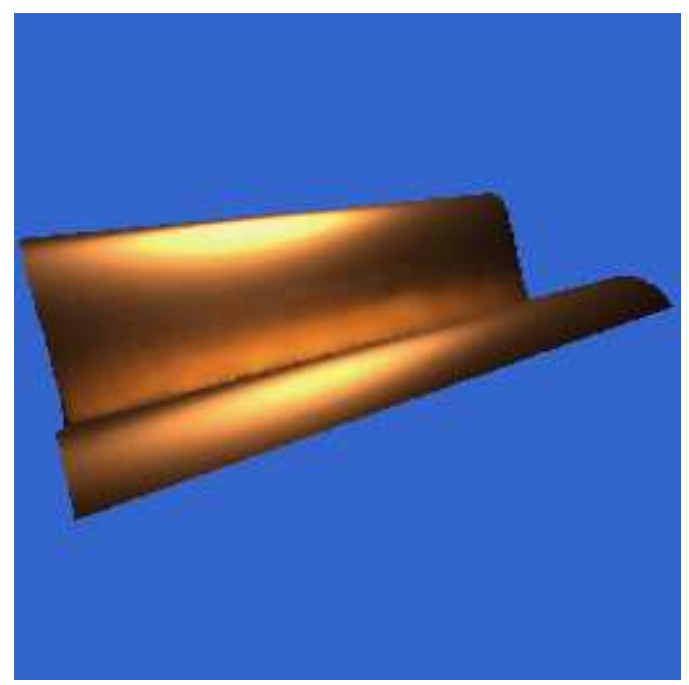

Figure 20: Scene 2

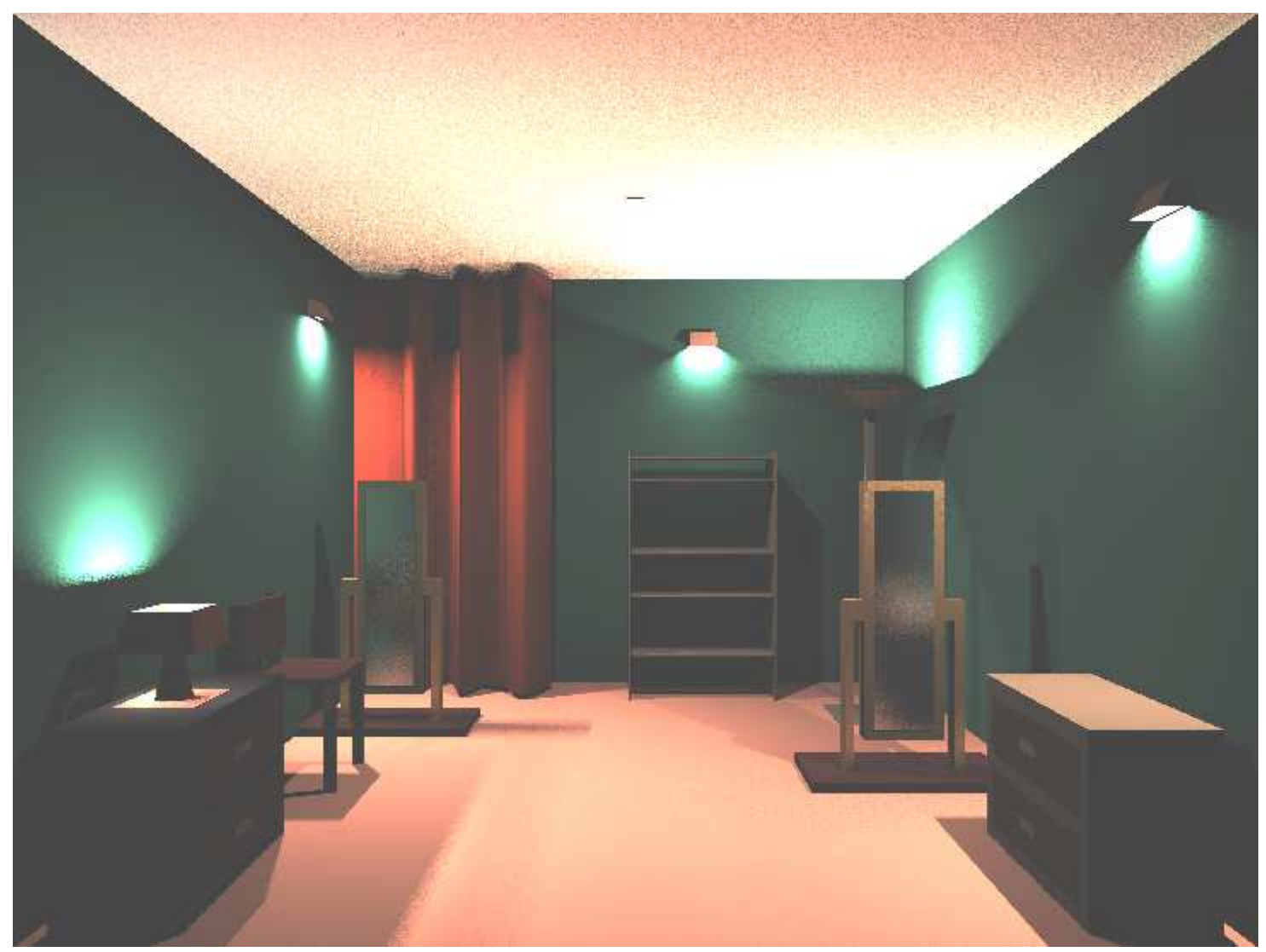

Figure 22: Scene 3 


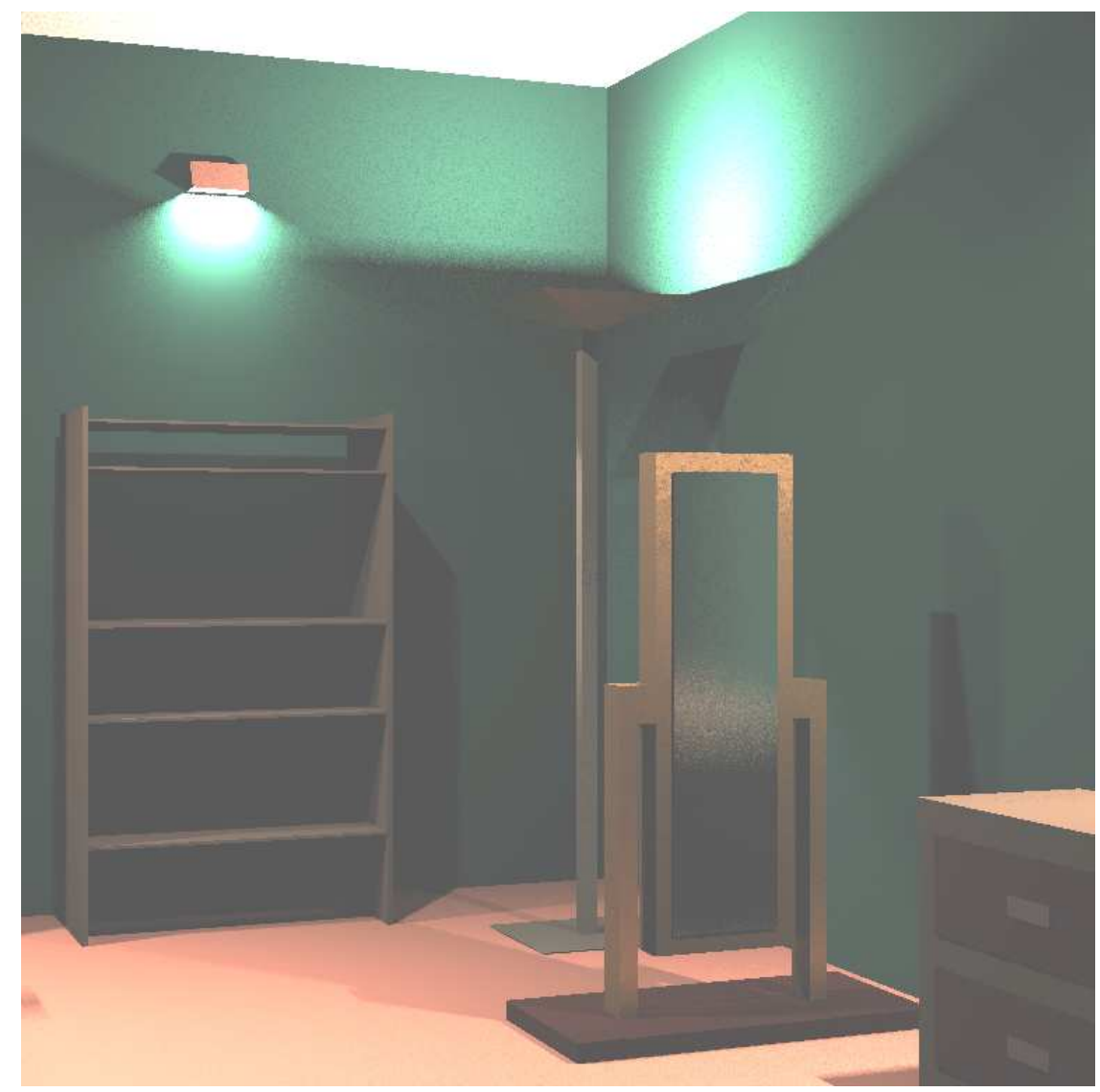

Figure 23: Scene 3 - Zoom 1

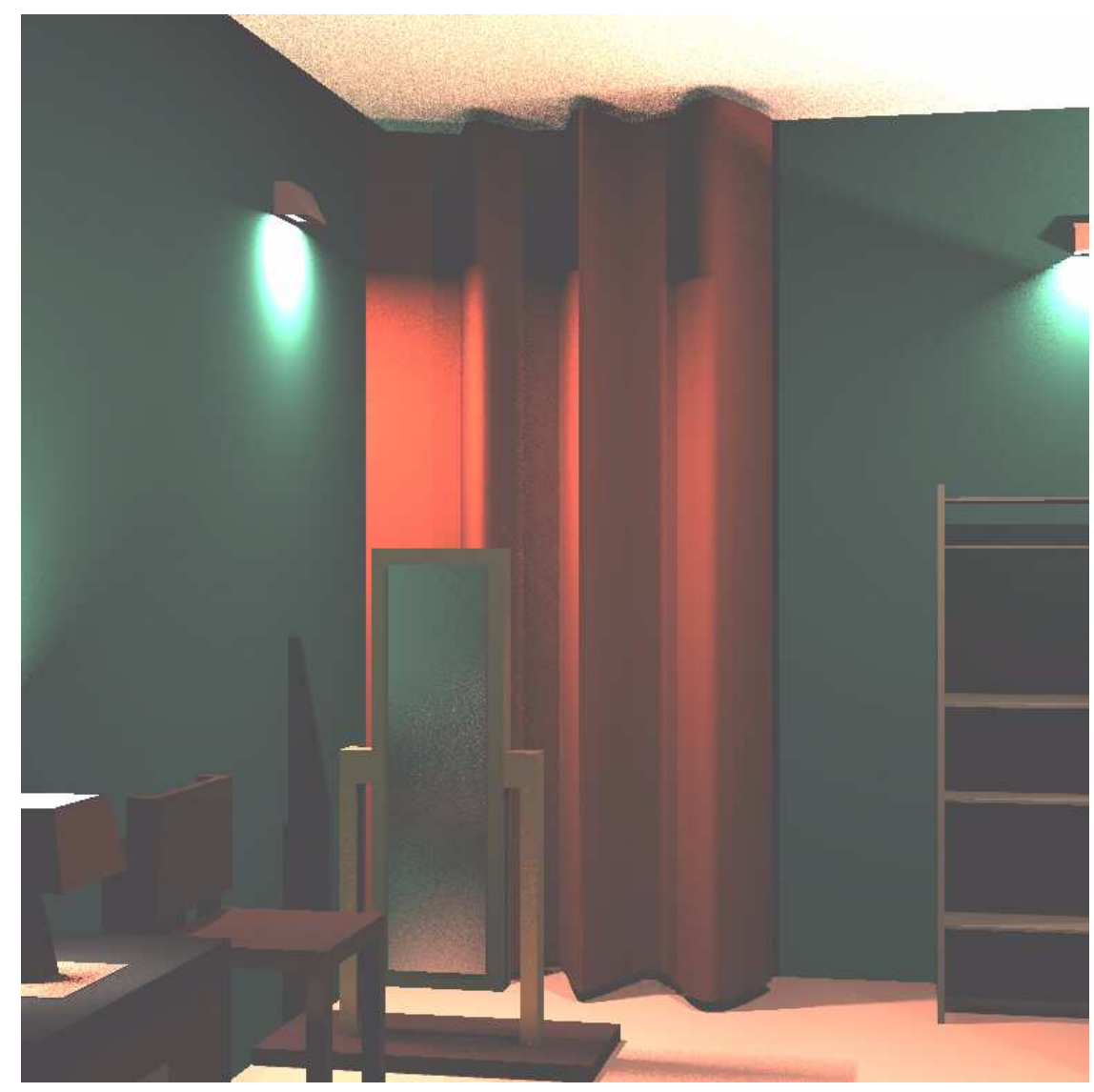

Figure 24: Scene 3 - Zoom 2 


\section{Conclusion}

The global illumination algorithm presented in this paper, at the same time, extends the three point transport algorithm ([ 2 ], [ 3 ]) to non-planar surfaces, and brings improvements regarding the computation time and memory size required. The used shooting process reduces the spatial complexity to $O\left(n+k^{2}\right)$ where $\mathrm{n}$ is the number of elements at the finest level of subdivision and $\mathrm{k}$ the number of input patches. The other improvements concern the refinement criterion, the push/pull operation and the view independence of the algorithm.

As extension, our algorithm can handle non-planar subdivisions with a few extra computation and memory storage thanks to the new interaction data structure (with direct and indirect links) and the associated management mechanism.

\section{References}

[ 1 ] Airey J. M. (1990) Increasing Update Rates in the Building Walkthrough System with Automatic Model-Space Subdivision And Potentially Visible Set Calculations. PhD Thesis of University of North Carolina.

[ 2 ] Aupperle L. (1993) Hierarchical algorithms for illumination. PhD thesis of Princeton university.

[ 3 ] Aupperle L., Hanrahan P. (1993) A hierarchical illumination algorithm for surfaces with glossy reflection. SIGGRAPH' 93 Proceedings, 155-162.

[ 4 ] Aupperle L., Hanrahan P. (1993) Importance and discrete three point transport. Proceedings of 4th Eurographics Workshop on Rendering, 85-94.

[ 5 ] Christensen P.H., Stollnitz E.J., Salesin D.H. DeRose T.D. (1994) Wavelet radiance. Proceedings of 5th Eurographics Workshop on Rendering, 287-302.

[6] Cohen M., Chen S.E., Wallace J.R., Greenberg D.P. (1988) A progressive refinement approach to fast radiosity image generation. SIGGRAPH '88 Proceedings, 75-84.

[ 7 ] Cook R., Porter T., Carpenter L. (1984) Distributed Ray tracing. SIGGRAPH' 84 Proceedings, 137-145.

[ 8 ] Hanrahan P., Salzman D., Aupperle L. (1991) A rapid hierarchical radiosity algorithm. SIGGRAPH '91 Proceedings, $197-$ 206.

[9] Immel D.S., Cohen M., Greenberg D.P. (1986) A radiosity method for non-diffuse environments. SIGGRAPH '86 Proceedings, 133-142.

[ 10 ] Kajiya J.T. (1986) The rendering equation. Computer Graphics 20, 143-150.

[11] Lafortune E.P., Willems D.Y. (1995) A 5D tree to reduce the variance of Monte Carlo ray tracing. Proceedings of 6th Eurographics Workshop on Rendering, 147-162.

[12] Pattanaik S., Bouatouch K. (1994) Haar wavelet : a solution to global illumination with general surface properties. Proceedings of 5th Eurographics Workshop on Rendering, 273-286.

[ 13 ] Pattanaik S., Mudur S. (1992) Computation of global illumination by Monte Carlo simulation of the particle model of light. Proceedings of 3rd Eurographics Workshop on Rendering, 71-83.

[ 14 ] Schröder P., Hanrahan P. (1994) Wavelet methods for radiance computations. Proceedings of 5th Eurographics Workshop on Rendering, 303-311.

[15] Sillion F., Puech C. (1989) A general two-pass method integrating specular and diffuse reflection. SIGGRAPH '89 Proceedings, 335-344.

[16] Sillion F.X., Arvo J.R., Westin S.H. Greenberg D.P. (1991) A global illumination solution for general reflectance distributions. SIGGRAPH' '91 Proceedings, 187-196.

[ 17 ] Teller S., Fowler C., Funkhouser T., Hanrahan P. (1994) Partitioning and ordering large radiosity computations. Computer Graphics Proceedings, Annual Conference Series, 443-450.

[18 ] Tellier P., Bouatouch K. (1991) Global illumination models: implementation issues. Proceedings of 2nd Eurographics Workshop on Rendering.

[19] Wallace J.R., Cohen M. F., Greenberg D.P. (1987) A two pass solution to the rendering equation: A synthesis of ray-tracing and radiosity methods. SIGGRAPH' 87 Proceedings, 311-320.

[ 20 ] Ward G.J., Rubinstein F.M., Clear R.D. (1988) A ray tracing solution for diffuse interreflection. SIGGRAPH '88 Proceedings, 85-92. 
Unit'e de recherche INRIA Lorraine, Technopôle de Nancy-Brabois, Campus scientifique,

615 rue du Jardin Botanique, BP 101, 54600 VILLERS LÈS NANCY

Unit'e de recherche INRIA Rennes, Irisa, Campus universitaire de Beaulieu, 35042 RENNES Cedex

Unit'e de recherche INRIA Rhône-Alpes, 655, avenue de l'Europe, 38330 MONTBONNOT ST MARTIN

Unit'e de recherche INRIA Rocquencourt, Domaine de Voluceau, Rocquencourt, BP 105, 78153 LE CHESNAY Cedex

Unit'e de recherche INRIA Sophia-Antipolis, 2004 route des Lucioles, BP 93, 06902 SOPHIA-ANTIPOLIS Cedex

Éditeur

INRIA, Domaine de Voluceau, Rocquencourt, BP 105, 78153 LE CHESNAY Cedex (France)

http://www.inria.fr

ISSN 0249-6399 